\title{
Effects of Dexmedetomidine Versus Ketorolac as Local Anesthetic Adjuvants on the Onset and Duration of Infraclavicular Brachial Plexus Block
}

\author{
Alireza Mirkheshti ${ }^{1}$; Asadollah Saadatniaki ${ }^{1}$; Alireza Salimi ${ }^{1}$; Alireza Manafi Rasi ${ }^{2}$; Elham \\ Memary ${ }^{1, *} ;$ Habiballah Yahyaei $^{1}$ \\ ${ }^{1}$ Anesthesiology Department, Shahid Beheshti University of Medical Sciences, Tehran, Iran \\ ${ }^{2}$ Orthopedic Department, Shahid Beheshti University of Medical Sciences, Tehran, Iran \\ *Corresponding author: Elham Memary, Anesthesiology Department, Shahid Beheshti University of Medical Sciences, Tehran, Iran. Tel: +98-2177567840, Fax: +98-2177567840, E-mail: \\ drmemary@gmail.com
}

Received: January 17, 2014; Revised: January 1,1970; Accepted: May 30, 2014

\begin{abstract}
Background: Infraclavicular brachial plexus block is an appropriate approach for distal arm and forearm surgeries. Local anesthetic adjuvant agents are used to improve the quality of nerve blocks. Dexmedetomidine and ketorolac are two different types of adjuvants, which have been used in some studies.

Objectives: The purpose of this study was to examine the effects of dexmedetomidine and ketorolac as local anesthetic adjuvants on the onset and duration of infraclavicular brachial plexus block under ultrasound guide technique.

Patients and Methods: In a clinical trial study, 111 ASA class I and II patients who were candidates for elective distal arm and forearm surgeries under ultrasound guided infraclavicular brachial plexus block divided into three 37 patient groups. In dexmedetomidine group, $25 \mathrm{~mL}$ of lidocaine $1.5 \%$ plus $4 \mathrm{ml}$ of normal saline and $100 \mathrm{mcg}$ of dexmedetomidine was injected. Ketorolac group received $25 \mathrm{~mL}$ of Lidocaine $1.5 \%$ plus $5 \mathrm{~mL}$ of ketorolac, and placebo group received $25 \mathrm{~mL}$ of lidocaine $1.5 \%$ plus $5 \mathrm{~mL}$ of normal saline as local anesthetic solution. Sensory and motor onset blocks, duration of sensory and motor blocks and first time to analgesic request and hemodynamic parameters were all recorded.

Results: There were no significant differences in sensory block onset between three groups $(\mathrm{P}=0.177)$. Motor block onset was statistically less in dexmedetomidine compared to ketorolac and placebo groups (both Ps $<0.001$ ). Sensory block duration in dexmedetomidine group was significantly longer than ketorolac and placebo groups (both Ps $<0.001$ ). Motor block duration in dexmedetomidine group was significantly longer than ketorolac and placebo groups (both Ps $<0.001$ ). Time to first analgesic request after the procedures was longer in ketorolac compared to dexmedetomidine and placebo groups $(\mathrm{P}=0.016, \mathrm{P}<0.001$ respectively), but it was longer in dexmedetomidine compared to placebo group $(\mathrm{P}=0.003)$. The differences of diastolic blood pressure in-between the 5th to 140th minutes after local anesthetic injection among the 3 groups were statistically significant and dexmedetomidine group shows the most reduction in diastolic blood pressure $(\mathrm{P}<0.001)$. Dexmedetomidine showed the lowest mean arterial pressure $(\mathrm{P}=0.016)$ and heart rate in dexmedetomidine group was significantly lower than ketorolac and placebo groups $(\mathrm{P}=0.043)$.

Conclusions: Our study showed that dexmedetomidine had better effects on sensory and motor block duration and motor block onset in comparison with ketorolac, as lidocaine adjuvants in infraclavicular brachial plexus block were present in both protocols. However, the first time to analgesic request by ketorolac was longer than dexmedetomidine.
\end{abstract}

Keywords: Ketorolac; Dexmedtomidine; Infraclavicular Brachial Plexus

\section{Background}

Infraclavicular brachial plexus block is a popular approach in accomplishing upper extremity anesthesia, which is well suited for distal arm and forearm surgeries. There are several techniques for infraclavicular nerve block by using nerve stimulation or ultrasound. Adjuvant agents are wide ranges of drugs, which is co-administered with local anesthetics to improve the speed of onset and quality or duration of nerve blocks. Dexmedetomidine is an agonist of $\alpha 2$ adrenergic receptors that in some trials, reduced the onset time and improved the duration of sensory and motor blocks (1-3); but in the other ones, it had no effect or even showing a delay in the onset and duration of sensory and motor blocks $(4,5)$. Eventhough,dexmedetomidine is a potential local anesthetic adjuvant with facilitative effects as a part of peripheral nerve blocks, the data regarding its complete safety on human beingsare insufficient (6). Ketorolac is a parenteral non-steroidal anti-inflammatory drug, which provides analgesia by inhibiting prostaglandin synthesis. It has been shown that ketorolac - as an adjuvant to local anesthetics - produces longer duration and better quality of analgesia during peripheral nerve block (7).

\section{Objectives}

In this study we compared dexmedetomidine and ketor-

Copyright ( 2014 , Iranian Society of Regional Anesthesia and Pain Medicine (ISRAPM); Published by KowsarCorp. This is an open-access article distributed under the terms of the Creative Commons Attribution License, which permits unrestricted use, distribution, and reproduction in any medium, provided the original work is properly cited. 
olac effects as local anesthetic adjuvants upon the onset and duration of sensory and motor nerve blocks under ultrasound guided infraclavicular brachial plexus block. Furthermore, we assessed hemodynamic parameters and time to first analgesic request of patients by distal arm and forearm surgeries.

\section{Patients and Methods}

After obtaining written informed consent from each patient and approval of institutional review board, 111 ASA classes I and II patients who were candidates for elective distal arm and forearm surgeries for less than 2.5 hours were selected and entered into our double-blinded randomized clinical trial. The exclusion criteria of our study comprised: any allergic reactions to NSAIDS, lidocaine and $\alpha 2$ agonists, all patients with hypertension, cardiac, hepatic or renal diseases, the patients who were under treatment of any $\alpha 2$ agonist or antagonist agents, pregnant women, drug abusers and psychiatric patients. Moreover, every patient who had anatomical or vascular abnormality in the upper extremity was excluded from our survey. By considering the statistical power of $95 \%$ and type one error of $\alpha=5 \%, 111$ patients were included and then divided into 3 groups by permuted block randomization, which every group consists of 37 patients as: Dexmedetomidine (D), Ketorolac (K) and Control (C) groups. All patients were monitored with electrocardiogram, pulse oximetry and noninvasive blood pressure monitoring at the time of their entrance to the operating room and throughout the procedures. They received midazolam $0.02 \mathrm{mg} / \mathrm{kg}$ and fentanyl $1 \mu \mathrm{g} / \mathrm{kg}$ intravenously as premedication.Infraclavicular brachial plexus blocks were performed by using ultrasound (SonoAce Pico, Samsung Medison Ultrasound machine, South Korea) and nerve stimulation (B Braun nerve stimulator, USA) techniques. Patients were in supine position by the head turning slightly to the opposite side of their blocks. The injured hand was placed on the patient's abdomen and the shoulder was moved downward as much as possible .The parasagittal linear probe (8-14 $\mathrm{MHz}$ ) by sterile sleeve was positioned near the coracoid process to identify the axillary artery. Then, the attempt was made to identify lateral, medial and posterior cords of brachial plexus, based on their positions relativeto the artery. After injecting of lidocaine $2 \%$ by a $2 \mathrm{~mL}$ syringe for anesthetizing the skin, a $50 \mathrm{~mm}$ and gauge 22 stimulating needle (B Braun, Germany) attached to nerve stimulator ( 0.8 to $0.5 \mathrm{~mA}, 0.1 \mathrm{~ms}$ ) was used to anesthetized each cord. In dexmedetomidine group, $30 \mathrm{~mL}$ of a solution containing $25 \mathrm{~mL}$ of lidocaine1.5\% plus $4 \mathrm{~mL}$ of normal saline and $100 \mu \mathrm{g}$ of dexmedetomidine (Precedex $200 \mu \mathrm{g} / 2 \mathrm{~mL}$, Hospira, USA) was injected by the amount of $10 \mathrm{~mL}$ for each cord. In ketorolac group, $30 \mathrm{~mL}$ of a solution containing $25 \mathrm{~mL}$ of lidocaine $1.5 \%$, plus $5 \mathrm{~mL}$ of ketorolac (ketorolac trometamol, $10 \mathrm{mg} / 1 \mathrm{~mL}$, Roche, UK) was injected as the mentioned amount. In control group, $30 \mathrm{~mL}$ of a solution containing $25 \mathrm{~mL}$ of lidocaine $1.5 \%$ plus $5 \mathrm{~mL}$ of normal saline was injected to anesthetize all three cords as $10 \mathrm{~mL}$ for every cord.

Sensory block was assessed by the loss to pinprick through a 22 gauge needle every 30 seconds. If the patient could not recognize the pinprick in all dermatomes related to musculocutaneous, median, ulnar and radial nerves, the time was recorded as sensory onset block. The duration between this time and the time of sense of pinprick in all related upper extremity dermatomes was recorded as duration of sensory block. All flexion and extension of elbow, wrist and fingers related to the function of musculocutaneous, median, ulnar and radial nerves were evaluated every 30 seconds after local anesthetic injection and the motor onset block was recorded when all mentioned movements were disappeared. The duration between this time and returning all flexion and extension movements was assessed as motor block duration. Systolic, diastolic and mean blood pressures, heart rate and saturation of Oxygen by pulse-oximetry were all recorded in the time of injection, 5th, 10th, 15th and 20th minutes and then every 10 minute till the end of operation. Meanwhile, time to first analgesic request according to visual analogue scale (VAS) more than 3 was recorded. Post operation pain was managed with repeated doses of morphine $(2 \mathrm{mg})$ to VAS 3 or less. Statistical analysis was performed by SPSS (version 21.0, IBM Co. Chicago, IL). Normal distribution of data was investigated by Kolmogorov-Smirnov test and Q-Q plot. To compare the results of the groups, we used Kruskall-Wallis test. In the last step, to compare the results throughout the study, we used GeneralizedLinear Mixed model (GLMM). Two by two comparisons of groups was adjusted for multiple comparisons by Bonferroni method. P values less than 0.05 were considered as statistically significant.

\section{Results}

As the consort diagram shows, 111 patients enrolled in the study, however 8 cases were excluded fromour survey due to incomplete nerve block and we had to consider general anesthesia as the second plan. Thus, data analysis was performed on 103 patients (Figure 1).

Characteristics of patients are shown in Table 1 and the sensory and motor block onsets and duration of sensory and motor blocks data all are depicted in Table 2 . No significant differences was seen in sensory block onset between three groups $(\mathrm{P}=0.177)$. Motor block onset was statistically shorter in dexmedetomidine, compared to ketorolac and placebo groups (both $\mathrm{P}<0.001$ ). Sensory block duration in dexmedetomidine group was significantly longer than ketorolac and placebo groups (all Ps < 0.001). Motor block duration in dexmedetomidine group was significantly longer than ketorolac and placebo groups (both Ps $<0.001$ ). Time to first analgesic request after the procedures according to VAS $>3$ was longer in ketorolac compared to dexmedetomidine and placebo groups ( $\mathrm{P}=0.016, \mathrm{P}<0.001$ respectively), but it was longer in dexmedetomidine compared to placebo group $(\mathrm{P}=$ 0.003) (Table 2). 
Mirkheshti A et al.

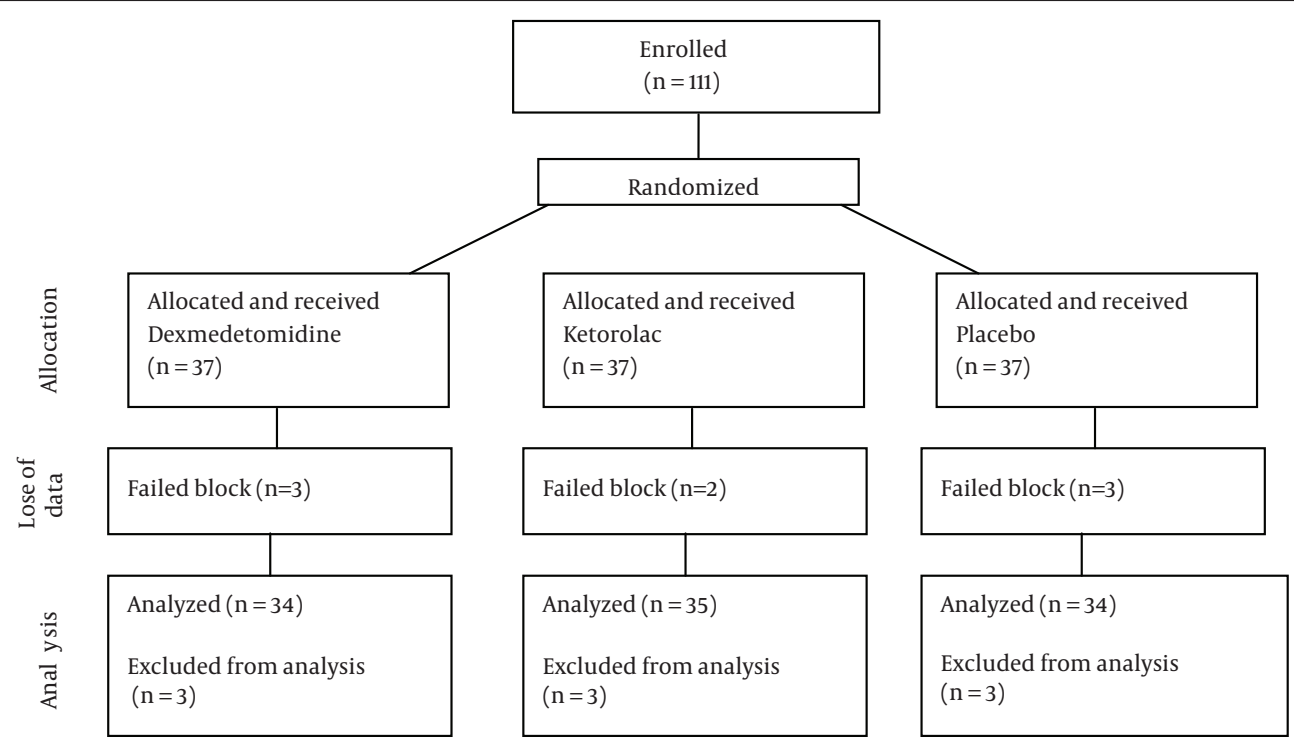

Figure 1. Consort Diagram Showing the Flow of Participants

Table 1. Characteristics of Patients in Three Groups ${ }^{a}$

\begin{tabular}{|c|c|c|c|c|c|}
\hline Parameter & Total & Dexmedetomidine & Ketorolac & Lidocaine & P Value \\
\hline Age, y & & & & & $0.990^{b}$ \\
\hline Mean \pm SD & $39 \pm 14$ & $39 \pm 14$ & $39 \pm 15$ & $39 \pm 15$ & \\
\hline Median (range) & $42(16-59)$ & $41(18-59)$ & $39(17-58)$ & $43(16-58)$ & \\
\hline Weight, kg & & & & & $0.363^{b}$ \\
\hline Mean \pm SD & $72 \pm 10$ & $72 \pm 9$ & $73 \pm 10$ & $70 \pm 10$ & \\
\hline Median (range) & $71(50-100)$ & $72(50-90)$ & $75(53-100)$ & $70(50-97)$ & \\
\hline Sex, No. (\%) & & & & & $0.104^{c}$ \\
\hline Male & $63(61.2)$ & $25(73.5)$ & $17(48.6)$ & $21(61.8)$ & \\
\hline Female & $40(38.8)$ & $9(26.5)$ & $18(51.4)$ & $13(38.2)$ & \\
\hline Systolic BP & & & & & $0.472^{b}$ \\
\hline Mean \pm SD & $130 \pm 18$ & $129 \pm 18$ & $132 \pm 19$ & $128 \pm 18$ & \\
\hline Median (range) & $127(97-189)$ & $126(103-189)$ & $131(97-166)$ & $122(102-169)$ & \\
\hline Diastolic BP & & & & & $0.035^{b}$ \\
\hline Mean \pm SD & $78.05 \pm 12.5$ & $73.94 \pm 10.82$ & $79.91 \pm 12.58$ & $80.29 \pm 13.28$ & \\
\hline Median (range) & $77(51-110)$ & $72(53-110)$ & $80(51-110)$ & $77(52-109)$ & \\
\hline MAP & & & & & $0.131^{b}$ \\
\hline Mean \pm SD & $95.29 \pm 13.08$ & $92.2 \pm 11.8$ & $97.33 \pm 13.26$ & $96.35 \pm 13.89$ & \\
\hline Median (range) & 93 (70 to 126$)$ & $88.5(72.33-124.67)$ & $96.33(73-123.33)$ & $93(70-126)$ & \\
\hline HR & & & & & $0.255^{b}$ \\
\hline Mean \pm SD & $83 \pm 12$ & $83 \pm 9$ & $81 \pm 14$ & $85 \pm 13$ & \\
\hline Median (range) & $84(57-130)$ & $86(60-97)$ & $80(57-130)$ & $86(66-109)$ & \\
\hline $\mathrm{SPO}_{2}$ & & & & & $0.091^{b}$ \\
\hline Mean \pm SD & $96 \pm 2$ & $97 \pm 2$ & $96 \pm 2$ & $97 \pm 2$ & \\
\hline Median(range) & $96(92-100)$ & $97(92-99)$ & $96(92-98)$ & $96(94-100)$ & \\
\hline
\end{tabular}

\footnotetext{
a Abbreviations: BP, blood pressure; MAP, mean arterial pressure; $\mathrm{HR}$, heart rate; $\mathrm{SPO}_{2}$, s pulse oximeter saturation of $\mathrm{O}_{2}$.

$\mathrm{b}$ Based on Kruskall-Wallis test.
}

c Based on Chi-Square test. 
Mirkheshti A et al.

\begin{tabular}{|c|c|c|c|c|}
\hline Parameter & Dexmedtomidine & Ketorolac & Lidocaine & P Value $^{a}$ \\
\hline Sensory block onset & & & & 0.177 \\
\hline Mean \pm SD & $8.68 \pm 3.57$ & $11.79 \pm 3.09$ & $11.91 \pm 4.66$ & \\
\hline Median (range) & $8(4-17)$ & $11(7-20)$ & $10.5(5-25)$ & \\
\hline Motor block onset & & & & $<0.001$ \\
\hline Mean \pm SD & $11.68 \pm 4.3$ & $15.94 \pm 3.67$ & $17.35 \pm 5.46$ & \\
\hline Median (range) & $10(7-25)$ & $16(9-27)$ & $17.5(6-30)$ & \\
\hline \multicolumn{5}{|l|}{ Sensory block duration } \\
\hline Mean \pm SD & $180 \pm 74$ & $132 \pm 54$ & $137 \pm 92$ & $<0.001$ \\
\hline Median (range) & 151 ( 41 to 372$)$ & $114(47-297)$ & $115(65-480)$ & \\
\hline Motor block duration & & & & $<0.001$ \\
\hline Mean \pm SD & $170 \pm 67$ & $109 \pm 50$ & $117 \pm 90$ & \\
\hline Median (range) & $140(80-360)$ & $98(40-292)$ & $106(70-450)$ & \\
\hline $\begin{array}{l}\text { first Time to analgesic } \\
\text { request }\end{array}$ & & & & $<0.001$ \\
\hline Mean \pm SD & $217 \pm 81$ & $315 \pm 145$ & $180 \pm 95$ & \\
\hline Median (range) & $188(47-402)$ & $291(107-620)$ & $162(91-510)$ & \\
\hline
\end{tabular}

$\mathrm{a}$ Based on Kruskall-Wallis test.

The difference of mean systolic blood pressure was not statistically significantamong the 3 groups during the procedures $(\mathrm{P}=0.476)$. The results of mixed regression model show that after considering the basic effects of mean diastolic blood pressure, the differences between diastolic pressure in 3 groups between 5 th to 140 th minutes after local anesthetic injection were statistically significant, Dexmedetomidine group shows the most reduction in diastolic blood pressure $(\mathrm{P}<0.001)$. The results of mixed regression model showed that every group had a decrease in mean arterial pressure (MAP), but dexmedetomidine group showed the lowest MAP during the procedures $(P=0.016)$ (Figure 2$)$. Heart rate in dexmedetomidine group was significantly lower than ketorolac and placebo groups, even by considering the basic effect of heart rates between 3 groups $(\mathrm{P}=0.043)$ (Figure 3 ). There were no side effects in each group.

\section{Discussion}

The purpose of the study was to assess the efficacy of dexmedetomidine and ketorolac as two different types of local anesthetic adjuvants in infraclavicular brachial plexus block. Our study demonstrated that the duration of motor and sensory blocks by dexmedetomidine were longer than Ketorolac. Our results about the increased duration of sensory and motor blocks by Dexmedetomidine as local anesthetic adjuvants are in agreement with the previous studies in different peripheral and neuraxial nerve blocks (8-14). Our results about ketorolac effects as local anesthetic adjuvant on duration of sensory and motor block support Budnyuk et al. study, in which ketorolac could not increase the duration of sensory and motor blocks when added to bupivacaine in brachial plexus

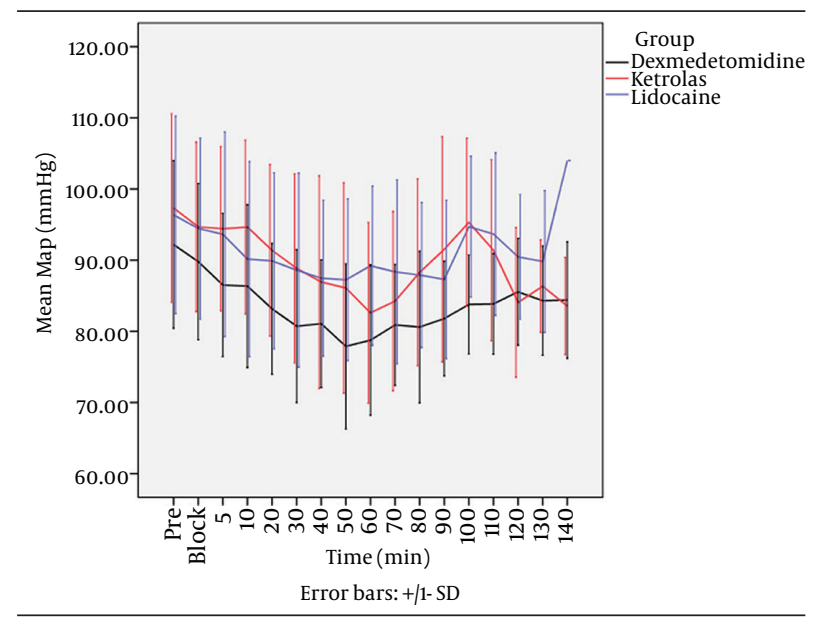

Figure 2. Mean Arterial Blood Pressure in Three Group

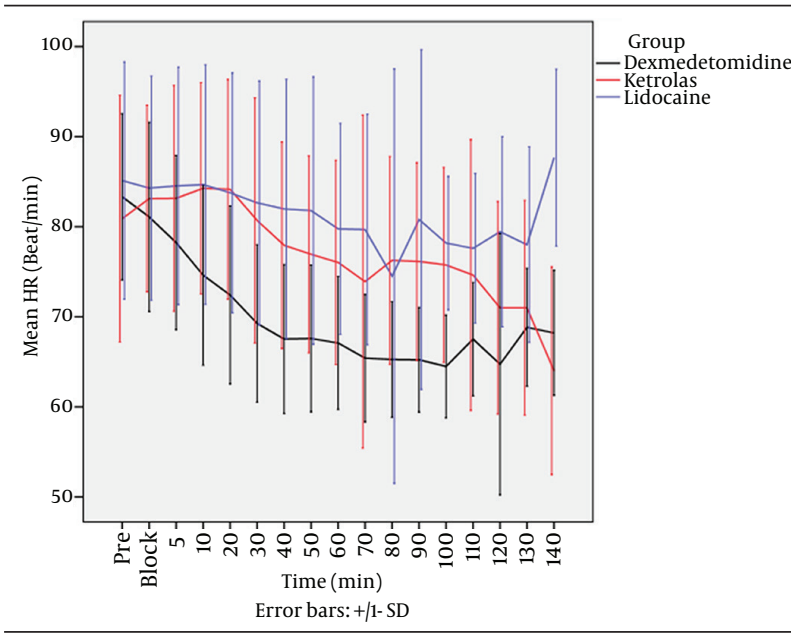

Figure 3. Mean Heart rate Between Three Groups 
block (15). The present study showed that ketorolac could not decrease sensory and motor block onset in infraclavicular brachial plexus block. The results about ketorolac on sensory and motor block onset provides more evidence for Budnyuk et al. study (15). However, our findings about sensory block onsets by dexmedetomidine are in disagreement with some studies $(3,11,12$, 14 ), which showed it could not decrease the onset time. One possible explanation for this disagreement on sensory block onset is that they performed their surveys by using bupivacaine as the main local anesthetic; but we used lidocaine as our main local anesthetic, which may cause different effects on sensory block onset. Our findings about motor block onset showed that dexmedetomidine decreased motor block onset compared to ketorolac and placebo, which are in agreement with Esmaoglu et al. (2) and Ammar et al. (11) showing that dexmedetomidine decrease motor onset time but the results are in disagreement with Kaygusuz study (9), in which dexmedetomidine did not decrease motor onset block. Our study showed that the time to first analgesic request by both ketorolac and dexmedetomidine increased. But, this increased time by ketorolac was more significant than dexmedetomidine. The results about the first time to analgesic request by both drugs support the previous studies $(13,14,16)$. The present study showed dexmedetomidine decreased mean arterial and diastolic blood pressures and heart rate during the procedures, which are in agreement with the other studies $(4,5,9,11)$. Our data about ketorolac effects also support the previous data (17).

The main limitation of this study was that it was not possible for us to evaluate neurologic complications caused by dexmedetomidine or ketorolac. In some studies dexmedetomidine produced deleterious effects on neural system (18), but in the others, the adverse effects have not seen yet $(14,19)$. We recommend further studies focusing in adverse effects of perineural injection of these drugs. We saw no immediate side effect in this study, but intravascular injection of local anesthetics and pneumothorax were reported in other studies $(20,21)$.

Our study showed that dexmedetomidine had better effects on sensory and motor block duration and motor block onset in comparison with ketorolac, as lidocaine adjuvants in infraclavicular brachial plexus block were present in both protocols. However, the first time to analgesic request by ketorolac was longer than dexmedetomidine.

\section{Acknowledgements}

The authors of this article would like to thank all participants that co-operated in this project; their dedication is deeply appreciated. We also would like to thank Dr Mehdi Yaseri, Ph.D of biostatics from department of epidemiology and biostatistics, Tehran University of Medical Sciences, Tehran, Iran for statistical analysis of the manuscript.

\section{Authors' Contributions}

Study concept and design, Dr Alireza Mirkheshti; Acquisition of data, Dr Habiballah Yahyaei and Dr Alireza Manafi Rasi; Drafting of the manuscript, Dr Elham Memary; Critical revision of the manuscript for important intellectual content, Dr Elham Memary; Study supervision, Dr Asadollah Saadat Niaki and Dr Alireza Salami.

\section{Funding/Support}

This project was financially supported by a grant from Clinical Research Development Unit, Imam Hossein Hospital, Shahid Beheshti University of Medical Sciences, Tehran, Iran.

\section{References}

1. Memis D, Turan A, Karamanlioglu B, Pamukcu Z, Kurt I. Adding dexmedetomidine to lidocaine for intravenous regional anesthesia. Anesth Analg. 2004;98(3):835-40.

2. Esmaoglu A, Yegenoglu F, Akin A, Turk CY. Dexmedetomidine added to levobupivacaine prolongs axillary brachial plexus block. Anesth Analg. 2010;111(6):1548-51.

3. Kanazi GE, Aouad MT, Jabbour-Khoury SI, Al Jazzar MD, Alameddine MM, Al-Yaman R, et al. Effect of low-dose dexmedetomidine or clonidine on the characteristics of bupivacaine spinal block. Acta Anaesthesiol Scand. 2006;50(2):222-7.

4. Gandhi R, Shah A, Patel I. Use of dexmedetomidine along with bupivacaine for brachial plexus block. National J Med Res. 2012;2(1):67-9.

5. Esmaoglu A, Mizrak A, Akin A, Turk Y, Boyaci A. Addition of dexmedetomidine to lidocaine for intravenous regional anaesthesia. Eur J Anaesthesiol. 2005;22(6):447-51.

6. Abdallah FW, Brull R. Facilitatory effects of perineural dexmedetomidine on neuraxial and peripheral nerve block: a systematic review and meta-analysis. BrJ Anaesth. 2013;110(6):915-25.

7. Reinhart DJ, Stagg KS, Walker KG, Wang WP, Parker CM, Jackson $\mathrm{HH}$, et al. Postoperative analgesia after peripheral nerve block for podiatric surgery: clinical efficacy and chemical stability of lidocaine alone versus lidocaine plus ketorolac. Reg Anesth Pain Med. 2000;25(5):506-13.

8. Fritsch G, Danninger T, Allerberger K, Tsodikov A, Felder TK, Kapeller M, et al. Dexmedetomidine added to ropivacaine extends the duration of interscalene brachial plexus blocks for elective shoulder surgery when compared with ropivacaine alone: a single-center, prospective, triple-blind, randomized controlled trial. Reg Anesth Pain Med. 2014;39(1):37-47.

9. Kaygusuz K, Kol IO, Duger C, Gursoy S, Ozturk H, Kayacan U, et al Effects of adding dexmedetomidine to levobupivacaine in axillary brachial plexus block. Curr Ther Res Clin Exp. 2012;73(3):103-11.

10. Aghdashi MM, Dehghan K, Shokohi S, Shafagh S. Unusually prolonged motor and sensory block following single injection ultrasound-guided infraclavicular block with bupivacaine and dexamethasone. Anesth Pain Med. 2013;3(2):260-2.

11. Ammar AS, Mahmoud KM. Ultrasound-guided single injection infraclavicular brachial plexus block using bupivacaine alone or combined with dexmedetomidine for pain control in upper limb surgery: A prospective randomized controlled trial. Saudi J Anaesth. 2012;6(2):109-14.

12. Al-Mustafa MM, Abu-Halaweh SA, Aloweidi AS, Murshidi MM Ammari BA, Awwad ZM, et al. Effect of dexmedetomidine added to spinal bupivacaine for urological procedures. Saudi Med J. 2009;30(3):365-70.

13. Eid H, Shafie M, Youssef H. Dose-related prolongation of hyperbaric bupivacaine spinal anesthesia by dexmedetomidine. Ain Shams J Anesthesiol. 2011;4:83-95.

14. Shukla D, Verma A, Agarwal A, Pandey HD, Tyagi C. Comparative 
study of intrathecal dexmedetomidine with intrathecal magnesium sulfate used as adjuvants to bupivacaine. J Anaesthesiol Clin Pharmacol. 2011;27(4):495-9.

15. Budnyuk, Qureshi, Marukhnyak, Tchuev, Basenko.. Use of Ketorolac as adjuvant in brachial plexus block with bupivacaine. EurJ Anaesthesiol. 2006;23:123.

16. Gupta R, Bogra J, Verma R, Kohli M, Kushwaha JK, Kumar S. Dexmedetomidine as an intrathecal adjuvant for postoperative analgesia. Indian J Anaesth. 2011;55(4):347-51.

17. Camu F, Van Overberge L, Bullingham R, Lloyd J. Hemodynamic effects of two intravenous doses of ketorolac tromethamine compared with morphine. Pharmacotherapy. 1990;10(6 Pt 2)):122S-6S.

18. Konakci S, Adanir T, Yilmaz G, Rezanko T. The efficacy and neurotoxicity of dexmedetomidine administered via the epidural route. EurJ Anaesthesiol. 2008;25(5):403-9.

19. Brummett CM, Norat MA, Palmisano JM, Lydic R. Perineural administration of dexmedetomidine in combination with bupivacaine enhances sensory and motor blockade in sciatic nerve block without inducing neurotoxicity in rat. Anesthesiology. 2008;109(3):502-11.

20. Shankar H, Simhan S. Transient neuronal injury followed by in travascular injection during an ultrasound guided stellate ganglion block. Anesth Pain Med. 2013;2(3):134-7.

21. Gauss A, Tugtekin I, Georgieff M, Dinse-Lambracht A, Keipke D, Gorsewski G. Incidence of clinically symptomatic pneumothorax in ultrasound-guided infraclavicular and supraclavicular brachial plexus block. Anaesthesia. 2014;69(4):327-36. 